\title{
Avaliação do uso de medicamentos por puérperas no pós-parto normal e cesárea em relação aos riscos sobre a lactação em um hospital do Rio Grande do Norte, Brasil
}

\author{
Evaluation of the use of medications by puerperas in the normal \\ and cesarean postpartum in relation to the risks on lactation in \\ a hospital of Rio Grande do Norte, Brazil
}

Recebido em: 23/03/2019 Aceito em: $\quad 07 / 06 / 2019$
João Beserra da SILVA ${ }^{\mathbf{1}}$; Almária Mariz BATISTA ${ }^{2}$ ${ }^{1}$ Escola Multicampi de Ciências Médicas, Universidade Federal do Rio Grande do Norte. Rua Dr. Carlindo Dantas, s/n, CEP 59300-000. Caicó, RN. Brasil. ${ }^{2}$ Departamento de Farmácia, Centro de Ciências da Saúde, Universidade Federal do Rio Grande do Norte. Av. General Gustavo Cordeiro de Farias, s/n, CEP 59010-180. Natal, RN. Brasil. E-mail:almariamariz@yahoo.com.br

\section{ABSTRACT}

This study aimed to evaluate the profile of the use of medicines by puerperal in the normal and cesarean postpartum of the Regional Hospital Dr Mariano Coelho, in the city of Currais Novos, RN, Brazil, about the risks on lactation. The study was observational and descriptive, based on the prescriptions filed by puerperal assisted during the year 2018. The potential risk of the drugs to the infant was evaluated, according to information from Micromedex, American Academy of Pediatrics, Brazilian Society of Pediatrics and the Brazilian Ministry of Health. Drugs were compiled by active principle and pharmacological class according to the Anatomical Therapeutic Chemical Code and subsequently analyzed using descriptive statistics. The study involved 101 postpartum women, the majority of whom underwent cesarean delivery $(61.39 \%)$. Most of the medicines used in the hospital were considered safe in breastfeeding or presenting minimal risks to infants. However, some bibliographic sources presented different classifications for the safety of medications during breastfeeding. Thus, prescribers and other health professionals involved in the process of orientation of puerperal should take into account the safety of medication use during this period.

Keywords: drug use; pharmaceutical services; postpartum; infant health; patient safety

\section{RESUMO}

O objetivo deste estudo foi avaliar o perfil do uso de medicamentos por puérperas no pós-parto normal e por cesareana do Hospital Regional Dr Mariano Coelho, Currais Novos, RN, Brasil, em relação aos riscos na lactação. Trata-se de estudo observacional, descritivo, a partir de prescrições arquivadas de puérperas assistidas durante o ano de 2018. Os medicamentos foram avaliados quanto ao risco para o lactente, conforme base de dados Micromedex, Academia Americana de Pediatria, Sociedade Brasileira de Pediatria e Ministério da Saúde. Os medicamentos foram compilados por princípio ativo e classe farmacológica de acordo com o sistema Anatomical Therapeutic Chemical Code e, posteriormente, analisados por estatística descritiva. Participaram do estudo 101 puérperas, a maioria submetida a parto cesárea $(61,39 \%)$, condição em que também houve predominância do número de medicamentos/prescrição $(7,61 \%)$ por cada puérpera, 
de um total de 592 prescritos. A maioria dos medicamentos utilizados no hospital eram compatíveis com a amamentação ou apresentavam riscos mínimos aos lactentes; no entanto, diferentes fontes bibliográficas podem abordar classificações diferentes para segurança do uso de medicamentos durante amamentação. Assim, prescritores e demais profissionais de saúde envolvidos no processo de orientação das puérperas devem manter-se embasados em fontes seguras sobre o uso de medicamentos durante este período.

Palavras-chave: uso de medicamentos; Assistência Farmacêutica; pós-parto; saúde do lactente; segurança do paciente

\section{INTRODUÇÃO}

O uso de medicamentos, tanto durante a gravidez quanto durante parto e puerpério, é de grande importância nos contextos clínico e biopsicossocial e deve ser sempre avaliado devido aos riscos e benefícios envolvidos. No pós-parto, o uso de medicamentos pode ocorrer, devido à necessidade de tratamento para combater infecções, depressão, além de doenças crônicas que requerem medicamentos de uso contínuo (1-6).

A passagem de fármacos do sangue ao leite materno ocorre por mecanismos que envolvem membranas biológicas, as quais possuem, em sua constituição, proteínas e fosfolípideos. Após atravessar o endotélio capilar, o fármaco passa ao interstício e atravessa a membrana basal das células alveolares do tecido mamário, de forma que proteínas e lipídeos da membrana exercem influência na velocidade de passagem e na concentração do fármaco no leite (7).

Assim, é importante avaliar a utilização de medicamentos por puérperas lactantes, pois estes podem influenciar diretamente no sucesso do aleitamento materno. Embora haja poucas contraindicações para amamentação, o uso de medicamentos pode ser entendido como uma barreira; logo, é importante fornecer orientações precisas e atualizadas, juntamente com apoio e incentivo, às mulheres durante a lactação $(8,9)$.

Fatores relacionados a uso de medicamentos durante amamentação podem determiner o desmame de forma desnecessária e danosa ao lactente, nutriz e família. Dentre os fatores citados estão a prescrição por médicos que não possuem conhecimento ou interesse sobre segurança do uso de medicamentos durante lactação, bulas de medicamentos comprovadamente seguros que recomendam interrupção da amamentação ou a não utilização de medicamentos durante lactação, e o receio materno de que medicamentos prescritos possam provocar efeitos adversos na criança (10-13).

Medicamentos, geralmente, são utilizados durante trabalho de parto para indução uterotônica (prostaglandinas e oxitocina), prevenção da hemorragia pós-parto (oxitocina e ergometrina) e controle da dor (opioides, óxido nitroso e anestésicos locais). Mulheres em trabalho de parto estão cada vez mais expostas a oxitocina exógena, cuja secreção endógena pulsátil está intimamente associada ao estabelecimento da amamentação. Há escassez de ensaios clínicos sobre a utilização de medicamentos intraparto e seu impacto no aleitamento materno como resultado primário, mas a análise de dados retrospectivos oferece alternativa para investigação de reações adversas insuspeitadas, como o impacto de fármacos no trabalho de parto (14-19).

Por outro lado, a decisão de amamentar em concomitância a tratamento medicamentoso com exposição anterior in útero difere da decisão para iniciar nova terapia no início do pós-parto. Da mesma forma, riscos de terapia de dose única ou tratamento a curto prazo podem diferir de riscos de terapia crônica $(10,20)$.

Estima-se que $83,3 \%$ a $97,6 \%$ das gestantes utilizam ao menos um medicamento durante gravidez ou período de internação para o parto. Portanto, prestar assistência adequada às necessidades de gestante e recém-nascido requer conhecimento sobre classificação de risco de medicamentos, para que estes riscos sejam avaliados considerando o período gestacional de maior risco quanto ao consumo de medicamentos; se seus metabólitos são capazes de atravessar a barreira placentária ou serem encontrados no leite materno; e se é realmente necessário o tratamento medicamentoso (21-24).

A dificuldade ética em realizar ensaios clínicos com gestantes e as dúvidas quanto aos riscos para 
o feto são argumentos que dificultam a necessidade de medicar mulheres grávidas. Sendo assim, destaca-se a importância de estudos, como revisões de literatura e abordagens epidemiológicas, sobre a utilização de medicamentos por gestantes que possam subsidiar a prescrição dos mesmos (25-27).

Diante desse contexto, este estudo teve como objetivo avaliar o perfil de uso de medicamentos por puérperas, pós-parto normal e cesárea, assistidas em um hospital regional, de forma a identificar os riscos de sua utilização para o lactente durante o período de amamentação.

\section{MÉTODO}

Trata-se de estudo observacional, descritivo, a partir de prescrições para puérperas assistidas no Hospital Regional Dr. Mariano Coelho, durante o período de julho a dezembro de 2018. Este é classificado como hospital geral, com 88 leitos, sendo 21 de Obstetrícia, 17 de Pediatria e 10 de Unidade de Cuidados Intermediários (UCI) neonatal. Está localizado em Currais Novos, RN, sendo referência para os municípios da Região Seridó do Rio Grande do Norte.

O estudo foi realizado no Setor de Obstetrícia do hospital, porém durante o período de estudo, este encontrava-se em reforma, então, somente duas enfermarias, que foram remanejadas para o Setor de Pediatria, encontravam-se disponíveis para acolhimento das puérperas, ambas com 4 leitos cada, totalizando 8 .

Constituíram sujeitos do estudo todas as gestantes submetidas a parto normal ou cesárea internadas nos setores de Clínica Obstétrica e Alojamento Conjunto, durante o ano de 2018, que aceitaram participar do estudo mediante assinatura do Termo de Consentimento Livre e Esclarecido (TCLE), uma vez que o Comitê de Ética e Pesquisa condicionou o acesso aos prontuários das puéperas à assinatura deste Termo pelas mesmas. Gestantes que abortaram, gestantes internadas para tratamento clínico e puérperas sem medicamentos prescritos constituíram critérios de exclusão do estudo. Quanto aos medicamentos analisados, foram considerados os prescritos logo após o pós-parto imediato até a alta hospitalar.

As variáveis do estudo foram idade, peso, tipo de parto, tempo de internação, medicamentos utilizados pós-parto imediato, quantidade de medicamentos por puérpera, problemas de saúde pré-existentes, complicações pré-parto, parto, pós-parto.

Os medicamentos foram avaliados conforme o risco à segurança do lactente de acordo com Ministério da Saúde (MS) (7), Sociedade Brasileira de Pediatria (SBP) (10), Academia Americana de Pediatria (AAP) (28) e a base de dados Micromedex (29). Em seguida, foram enquadrados em suas respectivas classes farmacológicas por meio do sistema Anatomical Therapeutic Chemical (ATC) (30).

O estudo foi aprovado pelo Comitê de Ética e Pesquisa da Faculdade de Ciências da Saúde do Trairi, Universidade Federal do Rio Grande do Norte (FACISA/UFRN), sob $\mathrm{n}^{\circ}$ CAAE 84943918.5.0000.5568.

Os dados foram analisados por meio de cálculo de frequência, porcentagem e média, utilizando software Excel.

\section{RESULTADOS E DISCUSSÃO}

Aceitaram participar do estudo 101 puérperas, sendo a maioria submetida a parto cesárea $(61,39 \%)$, condição em que também houve predominância do número de medicamentos/prescrição $(7,61 \%)$ por cada puérpera, de um total de 592 prescritos (Tabela 1). 
Tabela 1. Características das puérperas internadas em um hospital de Currais Novos, RN, durante o período de julho a dezembro de 2018

\begin{tabular}{|l|l|l|}
\hline \multicolumn{1}{|c|}{ VARIÁVEIS } & \multicolumn{1}{|c|}{ Parto Cesárea } & \multicolumn{1}{c|}{ Parto Normal } \\
\hline Quantidade de medicamentos prescritos & $472(79,73 \%)$ & $120(20,27 \%)$ \\
\hline$N^{0}$ medicamentos/puérpera & 7,61 & 3,07 \\
\hline Média de idade (anos) & $(\leq 13) 1,61 \%$ & $(\leq 15) 2,56 \%$ \\
\hline Média de peso (kg) & $(\geq 14-24) 29,03 \%$ & $(\geq 16-25) 48,72 \%$ \\
\hline Média de tempo de internação (dias) & $(\geq 25-34) 48,39 \%$ & $(\geq 36-44) 5,13 \%$ \\
\hline
\end{tabular}

A maioria das puérperas investigadas, submetidas a parto normal ou cesárea, não apresentava problemas de saúde pré-existentes nem complicações. Dentre os problemas de saúde pré-existentes, o mais frequente no parto cesárea foi hipertensão $(9,6 \%)$. Em relação ao parto normal, foi detectado 1 caso de hipertensão e 1 de hipotireoidismo (2,5\% cada), condições que ocorreram em uma mesma puérpera (Tabelas 2 e 3 ).
Com relação às complicações, ocorreram 13 (21\%) casos de urgência obstétrica no pré-parto cesárea, sendo que o tipo de urgência não foi especificado no prontuário, apenas estava descrito como urgência obstétrica, o que representa uma limitação do estudo. A segunda complicação mais frequente nos 2 tipos de parto foi pré-eclâmpsia, sendo $5(8 \%)$ casos em mulheres submetidas a parto cesárea e $3(7,7 \%)$ em submetidas a parto normal (Tabelas 2 e 3 ).

Tabela 2. Problemas de saúde pré-existentes e complicações em mulheres submetidas a parto cesárea em um hospital de Currais Novos, RN, durante o período de julho a dezembro de 2018

\begin{tabular}{|c|c|}
\hline VARIÁVEIS & VALOR ABSOLUTO/RELATIVO \\
\hline \multicolumn{2}{|c|}{ Problemas de saúde pré-existentes } \\
\hline Hipertensão & $6(9,6 \%)$ \\
\hline Diabetes & $2(3,2 \%)$ \\
\hline Hipotireoidismo & $1(1,6 \%)$ \\
\hline Problema de coagulação sanguínea & $1(1,6 \%)$ \\
\hline Nenhum & $52(84 \%)$ \\
\hline \multicolumn{2}{|c|}{ Complicações Pré-Parto, Parto e Pós-Parto } \\
\hline Urgência obstétrica no pré-parto & $13(21 \%)$ \\
\hline Pré-eclâmpsia & $5(8 \%)$ \\
\hline Desproporção cefalopélvica & $3(4,9 \%)$ \\
\hline Oligodrâmnio & $2(3,3 \%)$ \\
\hline Sofrimento fetal & $1(1,6 \%)$ \\
\hline Amniorrexe & $1(1,6 \%)$ \\
\hline Tromboflebite em membro inferior direito com varizes & $1(1,6 \%)$ \\
\hline Nenhuma & $36(58 \%)$ \\
\hline
\end{tabular}


Tabela 3. Problemas de saúde pré - existentes e complicações em mulheres submetidas a parto normal em um hospital de Currais Novos, RN, durante o período de julho a dezembro de 2018

\begin{tabular}{|c|c|}
\hline VARIÁVEIS & VALOR ABSOLUTO/RELATIVO \\
\hline \multicolumn{2}{|c|}{ Problemas de saúde pré-existentes } \\
\hline Hipertensão & $1(2,5 \%)$ \\
\hline Hipotireoidismo & $1(2,5 \%)$ \\
\hline Nenhuma & $37(95 \%)$ \\
\hline \multicolumn{2}{|c|}{ Complicações Pré-Parto, Parto e Pós-Parto } \\
\hline Pré-eclâmpsia & $3(7,7 \%)$ \\
\hline Nenhuma & $36(92,3 \%)$ \\
\hline
\end{tabular}

Os mesmos medicamentos prescritos no pós-parto normal também foram no pós-cesárea; contudo, este último, geralmente, demanda utilização de uma maior quantidade de medicamentos, além dos prescritos no pós-parto normal, principalmente, devido o parto cesárea ser um procedimento mais invasivo, requerendo um maior tempo de permanência das puérperas no ambiente hospitalar. Em ambas as condições, no geral, foi usado, como referência, o que é preconizado pela literatura concernente à classificação de risco de uso de medicamentos durante a amamentação $(7,10,28,29)$.

Outrossim, em Currais Novos, RN, antibacterianos para uso sistêmico, substâncias de sangue e soluções de perfusão, tramadol, dexametasona e prometazina foram prescritos, praticamente, apenas no pós-cesárea. Neste tipo de parto, foram prescritos $472(79,73 \%)$ medicamentos (7,61/puérpera), ao passo que no pós-normal foram prescritos 120 (20,27\%) medicamentos (3,07/puérpera).

Quanto às classes farmacológicas mais prescritas para as puérperas, predominaram antibacterianos para uso sistêmico $(35,3 \%)$ (Tabela 4$)$, sendo cefalotina, cefazolina e cefalexina (cefalosporinas de $1^{\mathrm{a}}$ geração) os mais prescritos. Neste contexto, é importante destacar o uso de benzilpenicilina e ciprofloxacino. Para o primeiro, conforme a classificação de risco do Micromedex (29), não é descartado risco aos recém-nascidos. Para o segundo, a classificação do Ministério da Saúde (7) aponta que seu uso deve ser criterioso.

Tabela 4. Classes terapêutica prescritas para puerpéras pós-parto em em um hospital de Currais Novos, RN, durante o período de julho a dezembro de 2018

\begin{tabular}{|l|c|}
\hline \multicolumn{1}{|c|}{ Classes Terapêuticas Prescritas } & $(\%)$ \\
\hline antibacterianos & 35,3 \\
\hline antihipertensivos & 29,4 \\
\hline substâncias de sangue - soluções de perfusão & 23,5 \\
\hline antiinflamatórios e antirreumáticos não - estereoides & 17,6 \\
\hline agentes para transtornos gastrointestinais funcionais & 17,6 \\
\hline analgésicos & 11,7 \\
\hline analgésicos opioides & 5,8 \\
\hline fármacos para distúrbios relacionados com o ácido & 5,8 \\
\hline antieméticos e antinauseantes & 5,8 \\
\hline antitrombóticos & 5,8 \\
\hline preparações de ferro e antianêmicos & 5,8 \\
\hline outros ginecológicos e uterotônicos & 5,8 \\
\hline hormônios pituitários, hipotalâmicos e análogos & 5,8 \\
\hline corticoides de uso sistêmico & 5,8 \\
\hline preparações para tireoide & 5,8 \\
\hline imunoglobulinas & 5,8 \\
\hline antihistamínicos para uso sistêmico & 5,8 \\
\hline
\end{tabular}


Geralmente, antibacterianos são prescritos para profilaxia de infecção pós-parto, principalmente, cefalosporinas. É importante ressaltar que esta classe foi prescrita apenas no pós-parto cesárea, o que pode ser justificado pelo fato de ser um tipo de parto que oferece maior risco de infecção puerperal e também devido a condições como enfermarias improvisadas; gestantes em tratamento clínico, inclusive, por infecções urinárias; ocupando as mesmas enfermarias que as puérperas. Assim, a maioria dos prescritores, por precaução, prescreveram estes medicamentos, os quais são preconizados para antibiotiprofilaxia pelas Diretrizes de Atenção à Gestante (31).

Em um estudo realizado com mulheres militares da região metropolitana de Belo Horizonte, MG, foi constatado que antibacterianos sistêmicos foram os mais utilizados $(16,9 \%)$ no período de amamentação (32), não sendo possível identificar o motivo. Estes medicamentos podem alterar a flora intestinal da criança, podendo causar diarreia e interferência na interpretação do resultado de culturas do lactente (7).

A segunda classe farmacológica mais prevalente foi antihipertensivos $(29,4 \%)$, sendo os medicamentos mais prescritos a hidralazina e o nifedipino, considerados de risco infantil mínimo e compatíveis com a amamentação conforme a literatura pesquisada. Em um estudo com mulheres tomando até $30 \mathrm{mg}$ de nifedipino diariamente foi mostrado que a dose infantil seria $8 \mu \mathrm{g} / \mathrm{kg} /$ dose, menos de $1,8 \%$ da dose pediátrica terapêutica, sugerindo compatibilidade com amamentação $(33,34)$. Quanto ao fármaco furosemida, foi relatado que este passa para o leite materno, inibindo a lactação $(35,36)$.Para a furosemida, Micromedex (29) apontou que o risco infantil não é descartado, a AAP (25) não apresentou qualquer descrição, a SBP (26) a descreve como possivelmente compatível, e o MS (7) recomenda que seu uso deve ser criterioso.

O amplo uso de antihipertensivos identificado na presente pesquisa pode ser explicado pelo fato de que, dentre os problemas de saúde pré-existentes estudados na pesquisa, a hipertensão apareceu com frequência de $6(9,6 \%)$ e diabetes $2(3,2 \%)$ dos casos em partos cesárea. Dentre as complicações no pré-parto, parto ou pós-parto, a pré-eclâmpsia no pré-parto apareceu como condição mais frequente $5(8 \%)$ entre os 62 pós-cesáreas estudados, sendo a hipertensão o problema de saúde pré-existente mais frequente $1(2,5 \%)$ e a pré-eclâmpsia no pré-parto $3(7,7 \%)$ como complicação mais frequente nos casos de pós-parto normal.

A classe farmacológica relacionada a substâncias para sangue-soluções de perfusão foi a terceira mais prevalente $(23,5 \%)$, sendo o soro glicosado $5 \%$ e soro fisiológico $0,9 \%$ os mais prescritos e apenas no pós-cesárea. Para esta classe não houve, na literatura pesquisada, recomendações acerca dos riscos à criança. É importante destacar que a ausência de informações não significa, necessariamente, risco descartado; indica apenas que não foram encontrados relatos na literatura (28).

Para a classe farmacológica anti-inflamatórios e antirreumáticos não-esteroidais nimesulida e diclofenaco foram os mais prescritos; escopolamina, metoclopramida e simeticona foram os agentes para transtornos gastrointestinais funcionais mais prescritos; e entre os analgésicos, prevaleceram dipirona e paracetamol.

A avaliação de informações de bulas dos 27 anti-inflamatórios comercializados no Brasil mostrou que apenas $51,9 \%$ disponibilizavam informações sobre a segurança de uso durante amamentação (36). A Micromedex (29) aponta para risco infantil não descartado para nimesulida e diclofenaco. À exceção deste, considerado compatível com a amamentação (7), as demais fontes consultadas $(7,10,28)$ não trazem classificação de risco para ambos. É importante destacar que a ausência de informações a respeito não significa, necessariamente, risco descartado, mas que não foram encontrados relatórios na literatura (28), o que reforça a importância de práticas de farmacovigilância para investigação e notificação de suspeitas de efeitos indesejáveis envolvendo o uso destes fármacos, particularmente, durante o período de amamentação.

Quanto ao contexto de agentes para transtornos gastrointestinais funcionais, é importante destacar escopolamina e simeticona. Para o primeiro, a classificação da AAP (28) não fez menção do efeito clínico sobre o infant; a SBP (10) definiu como prossivelmete compatível; e o MS (7), como de uso criterioso. Para o segundo, Micromedex (29) o 
classifica como risco infantil não descartado e as demais fontes utilizadas para classificação de risco não descreveram qualquer risco.

Quanto ao analgésico dipirona, Micromedex (29) apontou que o risco infantil não é descartado; e a SBP (10), como possivelmente perigoso. Em um estudo realizado em hospital de Duque de Caxias, RJ, que presta serviços de saúde pública às lactantes do município, $61,54 \%$ dos medicamentos prescritos eram analgésicos e anti-inflamatórios não esteroidais.

Outrossim, é importante destacar que, apesar de não comporem as classes farmacológicas mais prevalentes, dexametasona, tramadol e prometazina (prescritos apenas pós-cesárea) também oferecem riscos à criança. Para tramadol, Micromedex (29) apontou que o risco infantil foi demonstrado; SBP (26), como possivelmente compatível; e MS (7), como uso criterioso. Dexametasona foi classificada, via Micromedex (29), como risco infantil demonstrado; SBP (26) classificou como possivelmente compatível; MS (7) como uso criterioso; e AAP (25) não descreveu qualquer risco. Quanto à prometazina, Micromedex (29) apontou risco infantil não descartado; SBP (26) como possivelmente compatível; e AAP (25) nenhuma descrição.

Dexametasona e prometazina geralmente são utilizados durante pós-cesárea em caso de hipersensibilidade a opioides, utilizados no trabalho de parto para a analgesia de longa duração (37). Glicocorticoides apresentam propriedades analgésica e antiemética, além de efeitos anti-inflamatórios. $\mathrm{O}$ uso de dose única de dexametasona no perioperatório melhora o alívio da dor, comparado a placebo, em pacientes submetidos à cirurgia sob anestesia geral; contudo, por estar associado a níveis glicêmicos marginalmente maiores nas 24 horas de pós-operatório, deve ser evitado em pacientes com resistência insulínica $(37,38)$.

Nexte contexto, a atuação do farmacêutico e o conhecimento em Farmácia Clínica é imprescíndivel, pois atua no cuidado direto ao paciente, promove o uso racional de medicamentos e outras tecnologias em saúde, redefinindo sua prática a partir das necessidades dos pacientes. Desta forma, torna-se necessário que o farmacêutico esteja inserido no processo do cuidado às puérperas, participando do planejamento e da avaliação da farmacoterapia, prevenindo, identificando, avaliando e intervindo nos incidentes relacionados a medicamentos e a outros problemas relacionados à farmacoterapia, para que as puérperas utilizem de forma segura os medicamentos de que necessitam, contribuindo assim para segurança do paciente (39).

As limitações do estudo foram o fato de que durante a realização da pesquisa o Setor de Obstetrícia do hospital encontrava-se em reforma, e assim limitou o número de participantes; alguns parcientes não aceitaram participar da pesquisa, o que também limita o número de sujeitos estudados.

\section{CONCLUSÃO}

A maioria dos medicamentos utilizados no hospital eram compatíveis com a amamentação ou apresentavam riscos mínimos aos lactentes, o que contribui para menor possibilidade de ocorrência de efeitos indesejáveis e suspensão da amamentação. As classes farmacológicas eram condizentes com o perfil do hospital estudado.

A maioria das puérperas não apresentava qualquer problema de saúde pré-existente e complicações. No entanto, pode ser destacada a ocorrência de maior frequência de problemas hipertensivos, o que pode ter justificado o amplo uso de medicamentos anti-hipertensivos.

O estudo evidenciou que diferentes fontes bibliográficas podem abordar classificações diferentes para segurança do uso de medicamentos durante a amamentação. Dessa forma, é importante que prescritores e demais membros da equipe de saúde padronizem as fontes de informações utilizadas no que diz respeito à utilização de medicamentos durante a lactação, de maneira a evitar divergências de informações, incorporando aquelas que melhor possam contribuir para segurança do paciente por ocasião do uso de medicamentos.

Outrossim, é necessária a atuação do farmacêutico por meio da intensificação de ações de farmacovigilância e acompanhamento farmacoterapêutico a gestantes e puérperas, a fim de prevenir problemas relacionados a medicamentos (PRM) e, consequentemente, promover o uso racional de medicamentos. 
1 Costa JM, Rocha LM, Cristiane MS, Abelha LL, Almeida KCA. Análise das prescrições medicamentosas em uma maternidade de Belo Horizonte e classificação de riscos na gestação e amamentação. Rev Bras Farm Hosp Serv Saúde. 2012;3(1):32-36.

2 Perini E, Magalhães SMS, Noronha V. Consumo de medicamentos no período de internação para o parto. Rev. Saúde Pública. 2005;39(3):358-365. DOI: 10.1590/ S0034-89102005000300005.

3 Fragoso VMS, Silva ED, Mota JM. Lactentes e tratamento medicamentoso da rede pública de saúde. Rev Bras Prom Saúde. 2014;27(2):283-290. DOI: 10.5020/18061230.2014.p283.

4 Amir L, Pirotta M, Raval M. Breastfeeding - evidence based guidelines for the use of medicines. Australian Fam Physician. 2011;40(9):684-690.

5 Schirm E, Schwagermann M, Tobi H, de Jong-van den Berg LTW. Drug use during breastfeeding: a survey from the Netherlands. Eur J Clin Nutr. 2004;58(2):386-390. DOI: https://doi.org/10.1038/sj.ejen.1601799.

6 Jayawickrama H, Amir LH, Pirotta MV. GPs' decision making when prescribing medicines for breastfeeding women: content analysis of a survey. BMC Res Notes. 2010;3(82):1-9. DOI: 10.1186/1756-0500-3-82.

7 BRASIL. Ministério da Saúde. Amamentação e uso de medicamentos e outras substâncias: amamentação e uso de drogas. 2. ed. Brasília: Editora MS. 2014.

8 McClatchey AK, Shield A, Cheong LH, Ferguson SL, Cooper GM, Kyle GJ. Why does the need for medication become a barrier to breastfeeding? A narrative review. Women Birth. 2018;31(5):362-366. DOI: 10.1016/j. wombi.2017.12.004.

9 NHNRC. National Health and Medical Research Council. Infant feeding guidelines. Canberra: National Health and Medical Research Council. 2012.

10 SBP. Sociedade Brasileira de Pediatria. Uso de medicamentos e outras substâncias pela mulher durante a amamentação. 2017.

11 Hale TW, Rowe HE. Medications \& mothers' milk. 2017. [citado em 23 de março de 2019]. Disponível em: http:// www. medsmilk.com.

12 Chaves RG, Lamounier JA, César CC, Corradi MAL, Mello RP, Gontijo CM, Drumond JM. Amamentação e uso de antiinflamatórios não esteroides pela nutriz: informações científicas versus conteúdo em bulas de medicamentos comercializados no Brasil. Rev. Bras. Saúde Matern. Infant. 2006;6(3):269-276. DOI: 10.1590/ S1519-38292006000300002.

13 Ito S, Koren G, Einarson, TR. Maternal noncompliance with antibiotics during breastfeeding. Ann Pharmacother. 1993;27(1):40-42. DOI: 10.1177/106002809302700110.
14 Jordan S, Emery S, Watkins A, Evans JD, Storey M, Morgan $\mathrm{G}$. Associations of drugs routinely given in labour with breastfeeding at 48 hours: analysis of the Cardiff Births Survey. BJOG. 2009;116(12):1622-1632. DOI: 10.1111/j.1471-0528.2009.02256.x.

15 Winberg J. Mother and newborn baby: mutual regulation of physiology and behavior - a selective review. Dev Psychobiol. 2005;47:217-229. DOI: 10.1002/dev.20094.

16 Jordan S. Adverse drug reactions: reducing the burden of treatment. Nurs Stand. 2007;1:35-41.

17 Vandenbroucke JP. When are observational studies as credible as randomised trials? Lancet. 2004;363:17281731. DOI: 10.1016/S0140-6736(04)16261-2.

18 Kaufman D, Shapiro S. Epidemiological assessment of drug-induced disease. Lancet. 2000;356:1339-1343. DOI: $10.1016 / \mathrm{S} 0140-6736(00) 02826-9$.

19 Simpson J, Hannaford M. The contribution of cohort studies to prescribing research. J Clin Pharm Ther. 2002;27:151-156. DOI: 10.1046/j. 1365-2710.2002.00399.x.

20 AAP. American Academy of Pediatrics. The transfer of drugs and therapeutics into human breast milk: an update on selected topics. Pediatrics. 2013;132(3):796-809.

21 Góes AS. Fatores de risco e desfechos clínicos associados a problemas farmacoterapêuticos em mulheres puérperas com hipertensão na gestação. [Dissertação]. São Cristóvão. Universidade Federal de Sergipe. 2017.

22 Guérin A, Rieutordab A, Cuchec L Lupattellid A, Nordengde H. Médicaments et grossesse: une enquête menée auprès de 374 femmes en France. Therapies. 2016;71(6):613-623. DOI: 10.1016/j.therap.2016.04.005.

23 Andrade AM, Ramalho AA, Opitz SP, Martins FA, Koifman RJ. Farmacocinética e mecanismos de teratogenicidade dos medicamentos na gestação: uma revisão da literatura. Infarma - Ciênc Farm. 2017;29(2):100-107. DOI: 10.14450/2318-9312.v29.e2.a2017.pp100-107.

24 Koren G. Pharmacokinetics in pregnancy: clinical significance. J Popul Ther Clin Pharmacol. 2011;18(3):523527.

25 Steinkellner A, Chen W, Denison SE. Adherence to oral contraception in women on category X medications. Am J Med. 2010;123(10):929-934. DOI: 10.1016/j.amjmed.2010.05.009.

26 Schuler-Faccini L, Leite JCL, Sanserino MTV, Peres RM. Avaliação de teratógenos na população brasileira. Ciênc Saúde Colet. 2002;7(1):65-71. DOI: 10.1590/ S1413-81232002000100006.

27 FDA. Food and Drugs Administration. Prescription drug advertising: content and format for labeling of human prescription drugs. Rules and regulations. 1979. 
28 AAPCD. American Academy of Pediatrics Committee on Drugs. The transfer of drugs and other chemicals into human milk. Pediatrics. 2001;108(3):776-789.

29 THOMSON MICROMEDEX. 2014. [citado em 23 de março de 2018]. Disponível em: http://www.micromedexsolutions. com/micromedex2/librarian/.

30 WHO. World Health Organization. WHO Collaborating Centre for Drug Statistics Methodology. Anatomical Therapeutic Chemical (ATC): structure and principles. Geneva: WHO. 2013 [citado em 8 de janeiro de 2019]. Disponível em: http://www.whocc.no/atc/structure_and principles/.

31 BRASIL. Portaria n 306, de 28 de março de 2016. Regulamenta as diretrizes de atenção a gestante: a operação cesariana. Brasília: Ministério da Saúde; 2016.

32 Freitas TCSB, Lamounier JA, Chaves RG, Silva SC. Uso de medicamentos durante a gestação e a lactação em mulheres militares na região metropolitana de Belo Horizonte e sua associação com o tempo de aleitamento materno. Rev Med Minas Gerais. 2012;22(2): 158-165.

33 Bermas BL. Lactation and management of postpartum disease. Rheumatic Disease Clinics. 2017;43(2):249262. DOI: $10.1016 /$ j.rdc.2016.12.002.
34 Ehrenkranz RA, Ackerman BA, Hulse JD. Nifedipine transfer into human milk. J Pediatr. 1989;114:478-480. DOI: $10.1016 / \mathrm{S} 0022-3476(89) 80579-7$.

35 Ribeiro AS, Silva MV, Guerra PG, Saick KW, Uliana MP, Loss R. Risco potencial do uso de medicamentos durante a gravidez e a lactação. 2013;25(1):62-67. DOI: 10.14450/2318-9312.v25.e1.a2013.pp62-67.

36 Sutton CD, Carvalho B. Optimal pain management after cesarean delivery. Anesthesiology clinics. 2017;35(1):107-124. DOI: 10.1016/j.anclin.2016.09.010.

37 Ituk U, Thenuwara K. The effect of a single intraoperative dose of intravenous dexamethasone $8 \mathrm{mg}$ on post-cesarean delivery analgesia: a randomized controlled trial. Int J Obstet Anesth. 2018;35:57-63. DOI: 10.1016/j. ijoa.2018.03.008.

38 Waldron NH, Jones CA, Gan TJ, Allen TK, Habib AS. Impact of perioperative dexamethasone on postoperative analgesia and side-effects: systematic review and meta-analysis Br J Anaesth. 2013;110(2):191-200. DOI: 10.1093/bja/aes431

39 BRASIL. Resolução no 585, de 29 de agosto de 2013. Regulamenta as atribuições clínicas do farmacêutico e dá outras providências. Diário Oficial da União, p. 186, 2013 